\title{
Estimativas de Parâmetros Genéticos e Fenotípicos e Análise de Componentes Principais para Características de Crescimento na Raça Canchim
}

\author{
Arthur dos Santos Mascioli ${ }^{1}$, Lenira El Faro², Maurício Mello de Alencar ${ }^{3}$, Luiz Alberto Fries ${ }^{4}$, \\ Pedro Franklin Barbosa ${ }^{5}$
}

\begin{abstract}
RESUMO - Os objetivos deste trabalho foram obter estimativas de herdabilidade e de correlações genéticas, fenotípicas e de ambiente, para os ganhos diários de peso do nascimento à desmama (GND), da desmama aos 12 meses (GDA), dos 12 aos 18 meses (GASA) e dos 18 aos 24 meses (GSADA) de idade, e estimar componentes principais para essas características de crescimento na raça Canchim. Os dados

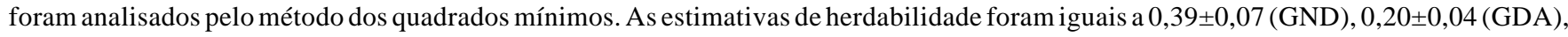

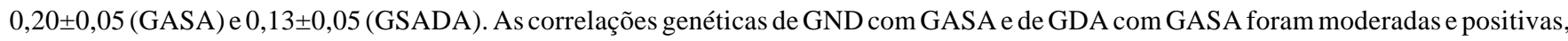
indicando associação genética favorável entre essas características. As correlações genéticas entre os ganhos de peso e os seus pesos adjacentes foram normalmente altas e positivas. Para os pesos, o primeiro componente principal explicou $74 \%$ da variação total e é um índice das características estudadas, cuja importância elevou-se de acordo com a idade do animal. O segundo componente explicou $14 \%$ da variação total e diferenciou animais com maiores pesos da desmama aos 18 meses de idade com aqueles de maiores pesos aos 24 meses de idade, ou seja, caracterizando animais com maior ou menor precocidade de crescimento e taxa de maturação precoce versus tardia. Para os ganhos de peso, o primeiro componente explicou 44\% da variação total e contrastou animais de maiores GDA e GSADA com animais de maiores GASA, ou seja, o ganho ocorrido nas épocas desfavoráveis com ganho durante a época favorável.
\end{abstract}

Palavras-chave: componentes principais, ganhos de peso, parâmetros genéticos, pesos

\section{Genetic and Phenotypic Parameters and Principal Components Analyses for Body Weight Gains in Canchim Cattle}

\begin{abstract}
The objectives of this study were to obtain estimates of heritability and genetic, phenotypic and environmental correlations for average daily gains from birth to weaning (DGBW), from weaning to 12 months (DGW12), from 12 to 18 months (DG1218) and from 18 to 24 months (DG1824) of age, and to estimate the principal components for these growth traits in Canchim cattle. The data were analyzed by the least square method. The heritability estimates were 0.39 0.07 (DGBW), $0.12 \pm 0.04$ (DGW12), 0.20 \pm 0.05 (DG1218) and 0.13 \pm 0.05 (DG1824). The genetic correlations between DGBW and DG1218, and DGW12 and DG1218 were moderated and positive, indicating favorable genetic association among these traits. The genetic correlations among body weight gains and adjacent body weights were, in general, high and positive. For body weights, the first principal component explained $74 \%$ of total variation, and it is an index of the studied traits, and the importance of each one increased as the animal aged. The second component explained $14 \%$ of the variation, and contrasts heavier animals at weaning and at 18 months with heavier animals at 24 months old, characterizing animals with higher or less precocity and precocious and late maturation rate. For body weight gains the first component explained 44\% of the total variation, and contrasts animals with higher DGW12 and DG1824 with animals with higher DG1218 or, in other words, contrasts the gains obtained during the less favorable season of the year (from weaning to one year of age and 18 to 24 months of age) with the gains obtained during the most favorable season of the year (from 12 to 24 months).
\end{abstract}

Key Words: genetic parameters, principal components, weights, weight gains

\section{Introdução}

A eficiência de produção em bovinos de corte depende de várias características e o melhoramento das características de importância econômica, por meio da seleção, depende do uso efetivo da variação genética existente nas mesmas. O conhecimento dos parâmetros genéticos das características e o entendimento das inter-relações entre elas é fundamental para o delineamento dos programas de seleção. Apesar de serem vários os trabalhos que reportam estimativas de parâmetros genéticos e fenotípicos para características de crescimento na raça Canchim (OLIVEIRA, 1979; PACKER , 1977; ALENCAR e

\footnotetext{
1 Estudante de pós-graduação - UNESP/Jaboticabal - Bolsista da FAPESP. E.mail: mascioli@fcav.unesp.br

2 Estudante de pós-graduação - UNESP/ Jaboticabal - Bolsista do CNPq. E.mail: lenira@fcav.unesp.br

3 Pesquisador da Embrapa/CPPSE, Caixa Postal 339, São Carlos - SP, 13560-970, Bolsista do CNPq.

4 GenSys Consultores Associados S/C Ltda. E.mail: fries@fcav.unesp.br

5 Pesquisador da EMBRAPA/CPPSE, Caixa Postal 339, São Carlos - SP, 13560-970. E.mail: pedro@cppse.embrapa.br
} 
BARBOSA, 1982; ALENCAR et al. 1993; FREITAS e VENCOVSKY, 1992; MASCIOLI et al., 1996; MASCIOLI et al., 1997), são poucos os trabalhos envolvendo ganhos de peso e as relações destes com os pesos (PACKER, 1977; OLIVEIRA, 1979; BARBOSA et al., 1980; MASCIOLI et al., 1997). É necessário, portanto, que esses parâmetros sejam estimados em amostra representativa da raça, para que programas de seleção possam ser elaborados.

De acordo com ROSO e FRIES (1995), o melhorista muitas vezes toma decisões, de forma intuitiva, selecionando animais em função de determinada característica, negligenciando seus componentes e as ligações existentes entre estes. Nesse contexto, a análise de componentes principais deve contribuir na interpretação das relações existentes entre as variáveis e, consequientemente, na tomada de decisões (BAKER et al., 1988; BARBOSA e SMITH, 1988; ROSO e FRIES, 1995).

Os objetivos deste estudo foram estimar parâmetros genéticos, fenotípicos e de ambiente para os ganhos diários de peso do nascimento à desmama (GND), da desmama aos 12 meses (GDA), dos 12 aos 18 meses (GASA) e dos 18 aos 24 meses (GSADA) de idade, e utilizar a análise de componentes principais para examinar a relação entre os pesos em diferentes idades e entre os ganhos de peso em animais da raça Canchim, verificando, assim, o grau de importância das características de desempenho em um programa de seleção.

\section{Material e Métodos}

Os dados utilizados neste trabalho são provenientes de cinco rebanhos da raça Canchim das fazendas Canchim (1), Guará (2), Baliza (3), Atlas (4) e Santa Úrsula (5), situadas nas regiões Central, Noroeste, Oeste, Leste e Sul do Estado de São Paulo. Foram estimadas as herdabilidades e as correlações genéticas, fenotípicas e de ambiente de GND, GDA, GASA e GSADA de, respectivamente, 2887, 3268, 2295, 1807 animais nascidos de 1982 a 1990, bem como as correlações entre esses ganhos de peso e os pesos ao nascimento $(\mathrm{PN})$, à desmama (PD, 240 dias), aos 12 (PA, 365 dias), 18 (PSA, 550 dias) e 24 (PDA, 730 dias) meses de idade. Os animais foram criados em regime de pastagens, formadas, principalmente, por braquiária (Brachiaria decumbens), braquiarão (Brachiaria brizantha), colonião (Panicum maximum) e andropogon (Andropogon gayanus), recebendo suplementação de minerais durante todo o ano. Em duas das cinco fazendas, os animais receberam suplementação de capim-napier (Pennisetum purpureum), durante as secas mais prolongadas (setembro e outubro), e de silagem de milho, após desmama. Apesar de haver nascimentos em todos os meses do ano, as fazendas adotavam uma ou duas estações de monta, com exceção da Fazenda Baliza, onde não havia estação de monta definida. Os acasalamentos endogâmicos eram evitados em todas as fazendas, com exceção da Fazenda Guará. Os animais receberam os cuidados sanitários comuns a cada região. Os pesos dos animais das fazendas Canchim e Atlas foram tomados por funcionários das próprias fazendas, enquanto os pesos nas outras fazendas, com exceção do peso ao nascimento, foram anotados por um funcionário da Fazenda Canchim. Como não era prática normal pesar animais ao nascer na Fazenda Guará, esse peso só foi disponível nas outras fazendas.

O dados foram analisados pelo método dos quadrados mínimos, cujos modelos matemáticos incluíram os efeitos fixos de fazenda, ano e mês de nascimento, sexo do animal e idade da vaca ao parto, além dos efeitos aleatórios de pai dentro de fazenda. Os parâmetros genéticos e fenotípicos foram estimados pelo método da correlação intra-classe entre meioirmãos paternos. As análises foram feitas utilizando-se o procedimento GLM (SAS, 1996).

Para análise dos componentes principais (CP), foram utilizadas 1116 observações de pesos e de ganhos de peso diários, utilizando-se o procedimento PRINCOMP contido no SAS (1996). As análises foram realizadas para os pesos e os ganhos de peso separadamente, utilizando-se três arquivos: no arquivo I as observações não foram ajustadas para nenhuma fonte de variação e a análise foi feita considerando-se os valores fenotípicos para as características; no arquivo II houve ajustes para os efeitos fixos de grupo contemporâneo (fazenda, ano, mês de nascimento e sexo do bezerro) e idade da vaca ao parto, e os componentes principais foram estimados utilizando-se os resíduos, e não os valores fenotípicos; e no arquivo III as análises foram as mesmas do arquivo II, mas as observações foram ajustadas também para os efeitos aleatórios de pai do animal dentro de fazenda.

\section{Resultados e Discussão}

O resumo das análises de variância dos ganhos de peso é apresentado na Tabela 1.

Verificou-se efeito significativo $(\mathrm{P}<0,01)$ do ano de nascimento sobre todos os ganhos de peso estudados. 
1656 Rev. bras. zootec.

Tabela 1 - Resumo das análises de variância de GND, GDA, GASA E GSADA

Table 1 - Analyses of variance of DGBW, DGW12, DG1218 and DG1824

\begin{tabular}{|c|c|c|c|c|c|}
\hline \multirow{3}{*}{$\begin{array}{l}\text { Fonte de variação } \\
\text { Sources of variation }\end{array}$} & \multirow{3}{*}{$\begin{array}{l}\text { Graus de liberdade } \\
\text { Degrees of freedom }\end{array}$} & \multicolumn{4}{|c|}{$\begin{array}{l}\text { Quadrados médios } \\
\text { Mean squares }\end{array}$} \\
\hline & & $\mathrm{GND}^{\mathrm{a}}$ & $\mathrm{GDA}^{\mathrm{a}}$ & GASA $^{a}$ & $\mathrm{GSADA}^{\mathrm{a}}$ \\
\hline & & $D G B W$ & $D G W 12$ & $D G 1218$ & $D G 1824$ \\
\hline$\overline{\text { Fazenda }(\mathrm{F})}$ & $4(3)^{b}$ & $0,602 * *$ & $3,223 * *$ & $1,312 * *$ & $0,163 * *$ \\
\hline \multicolumn{6}{|l|}{$\operatorname{Herd}(H)$} \\
\hline Ano de nascimento & $8(6)^{\mathrm{c}}$ & $0,244 * *$ & $0,800 * *$ & $0,465 * *$ & $1,087 * *$ \\
\hline \multicolumn{6}{|l|}{ Year of birth } \\
\hline Mês de nascimento & $11(10)^{\mathrm{c}}$ & $0,212 * *$ & $2,341 * *$ & $1,712 * *$ & $2,561 * *$ \\
\hline Idade da vaca & 11 & $0,085^{* *}$ & 0,071 & 0,040 & 0,035 \\
\hline \multicolumn{6}{|l|}{ Age of cow } \\
\hline Sexo do bezerro & 1 & $1,790 * *$ & $0,394 * *$ & $1,020 * *$ & $1,595 * *$ \\
\hline \multicolumn{6}{|l|}{ Sex of calf } \\
\hline $\mathrm{Pai} / \mathrm{F}$ & $102^{\mathrm{d}}$ & $0,045 * *$ & $0,109 * *$ & $0,053 * *$ & $0,060 * *$ \\
\hline \multicolumn{6}{|l|}{ Sire/H } \\
\hline Resíduo & $2751^{d}$ & 0,013 & 0,050 & 0,028 & 0,040 \\
\hline$\underline{\text { Error }}$ & & & & & \\
\hline \multirow{2}{*}{\multicolumn{6}{|c|}{$\begin{array}{l}\text { a GND, GDA, GASA, GSADA = ganhos diários de peso do nascimento à desmama, da desmama aos } 12 \text { meses, dos } 12 \\
\text { aos } 18 \text { meses e dos } 18 \text { aos } 24 \text { meses de idade, respectivamente. }\end{array}$}} \\
\hline & & & & & \\
\hline \multirow{2}{*}{\multicolumn{6}{|c|}{$\begin{array}{l}\text { b Graus de liberdade para GND entre parênteses. } \\
\text { c Graus de liberdade para GASA e GSADA entre parênteses. }\end{array}$}} \\
\hline \multirow{3}{*}{\multicolumn{6}{|c|}{$\begin{array}{l}\text { d Graus de liberdade de Pai/F iguais a } 124,115 \text { e } 102 \text { e do resíduo iguais a } 3108,2147 \text { e 1672, para GDA, GASA e GSADA, } \\
\text { respectivamente. }\end{array}$}} \\
\hline & & & & & \\
\hline${ }^{* *} \mathrm{P}<0,01$. & & & & & \\
\hline \multirow{2}{*}{\multicolumn{6}{|c|}{$\begin{array}{l}\text { a DGBW, DGW12, DG1218and DG1824= daily weight gains from birth to weaning, weaning to } 12 \text { months, } 12 \text { to } 18 \text { months and } 18 \text { to } 24 \text { months } \\
\text { of age, respectively. } \\
\text { b } D F \text { to DGBW within bracket. }\end{array}$}} \\
\hline & & & & & \\
\hline \multicolumn{6}{|c|}{ c DF to DG1218 and DG1824 within bracket. } \\
\hline - & & & & & \\
\hline
\end{tabular}

Observou-se grande variação nas médias de ano para ano, sem haver tendência de aumento ou redução nas mesmas com o passar dos anos. Os GND, GDA, GASA e GSADA variaram de $0,576(1988)$ a $0,718 \mathrm{~kg}$ (1990); 0,025 (1989) a 0,331 kg (1982); 0,201 (1988) a $0,344 \mathrm{~kg}(1983)$; e 0,192 (1982) a $0,469 \mathrm{~kg}(1988)$, respectivamente. As variações observadas nos ganhos diários de ano para ano, na maioria dos casos, podem ser atribuídas a oscilações na disponibilidade e qualidade das forrageiras, em conseqüência das condições climáticas, e aos manejos nutricionais, sanitários e reprodutivos aplicados aos animais em cada ano. $\mathrm{O}$ ambiente proporcionado às vacas, dentro de cada ano, teve influência indireta sobre o ganho de peso do nascimento à desmama. Já para os ganhos posteriores, o efeito do ambiente foi direto sobre o animal. $\mathrm{O}$ ano de nascimento pode, também, refletir mudanças na média do valor genético do rebanho, como conseqüência de um programa de seleção ou da introdução de touros e, ou, de vacas geneticamente superiores. Acrescente-se a isso o vigor híbrido resultante do acasalamento de animais de linhagens diferentes em determinados anos, como resultado da introdução de touros e vacas de rebanhos diferentes. Efeitos de ano de nascimento foram observados também por PACKER (1977) e OLIVEIRA (1979), mas não por MASCIOLI et. al. (1997), para o ganho diário do nascimento à desmama.

O mês de nascimento também influenciou significativamente $(\mathrm{P}<0,01)$ todos os ganhos de peso estudados (Tabela 1). Para o GND, o período de nascimento mais favorável foi o de abril a setembro, época fria do ano e de baixa precipitação pluviométrica, mais favorável à criação de bezerros, em razão da menor incidência de pragas e doenças. Para os GDA e GSADA, o período de nascimento mais favorável foi de fevereiro a junho, sendo que os períodos de ganhos coincidem com épocas de pastagens fartas (novembro a abril), fato que contribui para a alimentação do animal. Já para o GASA, o período de nascimento mais favorável foi de setembro a dezembro. Para os ganhos de peso após a desmama, a época de pesagem dos animais é que determina se o ganho é superior ou inferior. Assim, os animais nascidos no primeiro semestre apresentaram maiores GDA e GSADA, enquanto aqueles nascidos no segundo se- 
mestre apresentaram maior GASA, uma vez que passaram os últimos meses em pastagens com forragem farta e de boa qualidade. Os resultados obtidos neste trabalho corroboraram aqueles relatados por PACKER (1977), OLIVEIRA (1979), ALENCAR (1985) e MASCIOLI et. al. (1997).

O sexo do animal também apresentou efeito significativo $(\mathrm{P}<0,01)$ sobre todos os ganhos estudados (Tabela 1), sendo que os machos apresentaram maiores ganhos que as fêmeas, fato já observado por OLIVEIRA (1979), ALENCAR e BARBOSA (1982) e MASCIOLI et. al. (1997), na raça Canchim. As superioridades dos ganhos diários de peso a favor dos machos em relação ao ganho de peso das fêmeas representaram cerca de 8,$01 ; 11,55 ; 16,54$; e 20,13\%, para GND, GDA, GASA e GSADA, respectivamente, indicando aumento relativo na superioridade dos machos com o envelhecimento do animal. Essa tendência, além da diferença inerente ao sexo, pode ser causada por algum privilégio dado aos machos no manejo dentro das fazendas. Como os rebanhos estudados são rebanhos "puros", é provável que tenha havido maior retenção de fêmeas e maior descarte de machos após a desmama, o que ocasionaria aumento na diferença relativa entre os dois sexos, uma vez que os animais retidos no rebanho, geralmente, são os de melhor desempenho.

A idade da vaca ao parto apresentou efeito significativo $(\mathrm{P}<0,01)$ apenas sobre o GND (Tabela 1). Este resultado concorda com PACKER (1977) e MASCIOLI et al. (1997), os quais observaram que as vacas acima de cinco anos de idade, em geral, produziram bezerros que tiveram maior ganho de peso, possivelmente devido ao fato de as fêmeas terem atingido maturidade produtiva. Esse efeito sobre o GND era esperado, pois nesta fase o desenvolvimento do bezerro é praticamente dependente da mãe, o que não ocorre nos períodos posteriores estudados.

O efeito de fazenda influenciou significativamente todos os ganhos diários de peso estudados (Tabela 1). Verificou-se variação nos ganhos de peso dos animais de acordo com a fazenda, o que era esperado, devido às diferenças genéticas e nos manejos entre as fazendas. Os animais da fazenda 4 foram sempre os que apresentaram maiores ganhos de peso. Nesta fazenda, além de os animais serem criados em pastagens de excelente qualidade (pastos adubados), havia suplementação alimentar após a desmama. Esse rebanho era, também, um rebanho muito novo, com touros e vacas de várias procedências, o que deve ter resultado em vigor híbrido causado pelo acasalamentos de animais de linhagens diferentes. Esses efeitos genéticos e de meio ambiente certamente contribuíram para o excelente desempenho dos animais desta fazenda. O rebanho da fazenda número 1 é o mais antigo da raça e, por ser um rebanho de pesquisa, sempre foi intensamente manejado, apresentando o pior desempenho tanto para GND como para GDA. Entretanto, devido à maior intensidade de seleção realizada e, conseqüentemente, à maior taxa de descarte após a desmama, devem ter contribuído para melhores GASA e GSADA, sendo apenas inferiores aos animais da fazenda número 4. A fazenda número 3, apesar de ter estabelecido o rebanho há vários anos, ainda adquiria de outros rebanhos parte dos touros utilizados, o que poderia produzir algum vigor híbrido; entretanto, o manejo nutricional dos animais era pior que o dos outros rebanhos. O rebanho da fazenda número 2 também recebeu alguma suplementação alimentar durante a seca, mas é um rebanho fechado por vários anos, apresentando animais com desempenho próximo da média geral.

As estimativas de herdabilidade e de correlações genéticas, fenotípicas e de ambiente para os ganhos de peso são apresentadas na Tabela 2. Observaram-se valores baixos de herdabilidade para GDA $(0,20 \pm 0,04), \operatorname{GASA}(0,20 \pm 0,05)$ e $\operatorname{GSADA}(0,13 \pm 0,05)$ e valor moderado para GND $(0,39 \pm 0,07)$. O valor para GND está dentro da amplitude daqueles estimados por PACKER (1977), OLIVEIRA (1979), BARBOSA et al. (1980), SILVA et al. (1982) e MASCIOLI et. al. (1997). Estes resultados indicam que GND é uma característica que responde bem à seleção, enquanto GDA, GASA e GSADA devem apresentar menor resposta.

As correlações genéticas de GND com GASA $(0,44 \pm 0,15)$ e de GDA com GASA $(0,57 \pm 0,20)$ foram moderadas e positivas e aquelas de GDA com GSADA $(-0,49 \pm 0,25)$ e de GASA com GSADA $(-0,56 \pm 0,51)$, moderadas e negativas, indicando associação genética favorável de GND e GDA com GASA e antagônica quando GSADA é envolvido, ou seja, os resultados sugerem que a seleção para aumentar GND e, ou, GDA, resultará em maior GASA e menor GSADA. Cabe ressaltar os elevados erros-padrão das correlações, principalmente entre GASA e GSADA.

Na raça Canchim, poucos são os trabalhos que relatam estimativas de correlações genéticas, fenotípicas e de ambiente entre ganhos de peso posteriores à desmama. As correlações genéticas 
1658 Rev. bras. zootec.

Tabela 2 - Estimativas de herdabilidade $\left(h^{2}\right)$ e correlações genéticas $\left(r_{g}\right)$, fenotípicas $\left(r_{p}\right)$ e de ambiente $\left(r_{e}\right)$ de GND, GDA, GASA e GSADA

Table 2 - Estimates of herdability and genetic, phenotypic and environmental correlations for DGBW, DGW12, DG1218 and DG1824

\begin{tabular}{|c|c|c|c|c|}
\hline \multirow{3}{*}{$\begin{array}{l}\text { Características } \\
\text { Traits }\end{array}$} & \multicolumn{4}{|c|}{ Características $^{1}$} \\
\hline & GND & GDA & GASA & GSADA \\
\hline & $D G B W$ & $D G W 12$ & $D G 1218$ & $D G 1824$ \\
\hline$\overline{\mathrm{GND}}$ & $\mathbf{0 , 3 9} \pm \mathbf{0 , 0 7}$ & $0,23 \pm 0,16$ & $0,44 \pm 0,15$ & $-0,19 \pm 0,19$ \\
\hline GDA & $\begin{array}{l}-0,16 \\
-0,30\end{array}$ & $0,20 \pm 0,04$ & $0,57 \pm 0,20$ & $-0,49 \pm 0,25$ \\
\hline GASA & $\begin{array}{r}0,03 \\
-0,18\end{array}$ & $\begin{array}{l}-0,19 \\
-0,38\end{array}$ & $0,20 \pm 0,05$ & $-0,56 \pm 0,51$ \\
\hline GSADA & $\begin{array}{r}-0,04 \\
0,03\end{array}$ & $\begin{array}{c}-0,018 \\
0,09\end{array}$ & $\begin{array}{l}-0,27 \\
-0,26\end{array}$ & $0,13 \pm 0,05$ \\
\hline $\begin{array}{l}1 \text { GND, GDA, GA } \\
\text { aos } 12 \text { meses, } \\
\text { DGW12, DG1218, } \\
\text { and } 18 \text { to } 24 \text { mon } \\
2 \mathrm{~h}^{2} \text { na diagonal, } r \\
\text { and } r_{p} \text { and } r_{e} \text { bel }\end{array}$ & $\begin{array}{l}\text { ADA = ganh } \\
\text { aos } 18 \text { meses } \\
4=\text { daily weigh } \\
\text { ge, respectivel } \\
\text { da diagonal, }\end{array}$ & $\begin{array}{l}\text { s de peso do } \\
8 \text { aos } 24 \text { mes } \\
\text { om birth to wean }\end{array}$ & $\begin{array}{l}\text { nento à des } \\
\text { idade, resp } \\
\text { eaning to } 12\end{array}$ & $\begin{array}{l}\text { da desmama } \\
\text { ente. (DGBW, } \\
12 \text { to } 18 \text { months } \\
\text { ve the diagonal, }\end{array}$ \\
\hline
\end{tabular}

obtidas neste estudo para GND foram semelhantes àquelas obtidas por OLIVEIRA (1979), diferindo, entretanto, para os demais ganhos. Já os resultados de BARBOSA et al. (1982) foram bem diferentes, quando comparados com os obtidos neste estudo.

As correlações fenotípicas e de ambiente entre os ganhos de peso foram de baixa magnitude (Tabela 2), indicando baixa associação fenotípica e genotípica não-aditiva e, ou, de ambiente entre eles.

As correlações genéticas entre os ganhos de peso e os seus pesos adjacentes, apresentadas na Tabela 3, foram altas para PD e GND $(0,97 \pm 0,01)$, PD e GDA $(0,73 \pm 0,07)$, PA e GDA $(0,74 \pm 0,07)$, PA e GASA $(0,66 \pm 0,15)$ e PSA e GASA $(0,85 \pm 0,07)$, indicando elevada associação entre os pesos da desmama aos 18 meses com os ganhos entre estas idades. As correlações envolvendo GSADA foram baixas. As correlações fenotípicas em geral sugerem que os animais que apresentam ganhos de peso acima da média devem manter parte dessas vantagens nos seus pesos adjacentes. As correlações de ambiente sugerem que existe alguma associação genética não-aditiva e, ou, de ambiente entre as características estudadas.

$\mathrm{Na}$ Tabela 4 são apresentados os coeficientes dos componentes principais (CP). Cabe ressaltar que os componentes principais para cada arquivo são ortogonais entre si, indicando independência entre eles. Observa-se que os dois primeiros $\mathrm{CP}$ foram responsáveis por mais de $75 \%$ da variação total, tanto para os ganhos como para os pesos. Observa-se, também, que os componentes foram muito semelhantes para os três arquivos utilizados, para ambos os tipos de características. Este fato é interessante, uma vez que no arquivo 1 a variação inclui toda a variância genética e não-genética; no

Tabela 3 - Estimativas de correlações genéticas $\left(r_{g}\right)$, fenotípicas $\left(r_{p}\right)$ e de ambiente $\left(r_{e}\right)$ entre os ganhos de peso e os pesos adjacentes

Table 3 - Estimates of genetic, phenotypic and environmental correlations among the body weight gains and the adjacent body weights

\begin{tabular}{lccr}
\hline $\begin{array}{l}\text { Características } \\
\text { Traits }\end{array}$ & $\mathrm{r}_{\mathrm{g}}$ & $\mathrm{r}_{\mathrm{p}}$ & $\mathrm{r}_{\mathrm{e}}$ \\
\hline $\begin{array}{l}\text { PNe GND } \\
B W \text { and } D G B W \\
\text { PD e GND }\end{array}$ & $0,36 \pm 0,13$ & 0,11 & $-0,04$ \\
$\begin{array}{l}W W \text { and } D G B W \\
\text { PD e GDA }\end{array}$ & $0,97 \pm 0,01$ & 0,95 & 0,93 \\
$W W$ and $D G W 12$ & $0,73 \pm 0,07$ & 0,49 & 0,50 \\
$\begin{array}{l}\text { PA e GDA } \\
W 12 \text { and } D G W 12\end{array}$ & $0,74 \pm 0,07$ & 0,50 & 0,42 \\
PA e GASA & $0,66 \pm 0,15$ & $-0,13$ & $-0,47$ \\
$\begin{array}{l}W 12 \text { and } D G 1218 \\
\text { PSA e GASA }\end{array}$ & $0,85 \pm 0,07$ & 0,57 & 0,50 \\
$\begin{array}{l}W 18 \text { and } D G 1218 \\
\text { PSA e GSADA }\end{array}$ & $-0,26 \pm 0,21$ & $-0,21$ & $-0,21$ \\
$\begin{array}{l}W 18 \text { and } D G 1824 \\
\text { PDA e GSADA }\end{array}$ & $0,21 \pm 0,23$ & 0,52 & 0,59 \\
$W 24$ and $D G 1824$ & & & \\
\hline
\end{tabular}

1 PN, PD, PA, PSA, PDA, GND, GDA, GASA e GSADA são os pesos ao nascimento, à desmama e aos 12, 18, 24 meses de idade, ganhos diários de peso do nascimento à desmama, da desmama aos 12 meses, dos 12 aos 18 meses e dos 18 aos 24 meses de idade, respectivamente. (BW, WW, W12, W18, W24, DGBW, DGW12, DG1218, DG1824 = birth, weaning, 12, 18and 24 months of age weights, daily weight gains from birth to weaning, weaning to 12 months, 12 to 18 months and 18 to 24 months of age, respectively). 
MASCIOLI et al.

Tabela 4 - Coeficientes dos componentes principais para os pesos e ganhos de peso, de acordo com o arquivo de dados Table 4 - Coefficients of the principal components to the body weights and body weight gain, according to the data set

\begin{tabular}{|c|c|c|c|c|c|c|c|c|c|c|c|c|c|c|c|}
\hline \multirow{3}{*}{$\begin{array}{l}\text { Característica }^{1} \\
\text { Trait }\end{array}$} & \multicolumn{15}{|c|}{$\begin{array}{l}\text { Componentes principais } \\
\text { Principal components }\end{array}$} \\
\hline & \multicolumn{5}{|c|}{$\begin{array}{l}\text { Arquivo I } \\
\text { Data set I }\end{array}$} & \multicolumn{5}{|c|}{$\begin{array}{c}\text { Arquivo II } \\
\text { Data set II }\end{array}$} & \multicolumn{5}{|c|}{$\begin{array}{l}\text { Arquivo III } \\
\text { Data set III }\end{array}$} \\
\hline & 1 & 2 & 3 & 4 & 5 & 1 & 2 & 3 & 4 & 5 & 1 & 2 & 3 & 4 & 5 \\
\hline $\begin{array}{l}\mathrm{PN} \\
B W\end{array}$ & 0,01 & 10,03 & 0,01 & $-0,01$ & 1,00 & 0,03 & 0,00 & 0,02 & 0,02 & 1,00 & 0,03 & 0,00 & 0,01 & 0,03 & 1,00 \\
\hline $\begin{array}{l}\text { PD } \\
W W\end{array}$ & 0,27 & $\begin{array}{ll}7 & 0,47\end{array}$ & 0,42 & 0,73 & $-0,01$ & 0,34 & 0,55 & 0,40 & 0,65 & $-0,03$ & 0,32 & 0,53 & 0,40 & 0,67 & $-0,03$ \\
\hline $\begin{array}{l}\text { PA } \\
W 12\end{array}$ & 0,39 & $\begin{array}{ll}9 & 0,43\end{array}$ & 0,45 & $-0,67$ & $-0,03$ & 0,41 & 0,44 & 0,26 & $-0,75$ & 0,00 & 0,40 & 0,46 & 0,30 & $-0,73$ & 0,00 \\
\hline $\begin{array}{l}\text { PSA } \\
W 18\end{array}$ & 0,52 & $2 \quad 0,36$ & $-0,77$ & 0,02 & $-0,01$ & 0,53 & 0,16 & $-0,82$ & 0,10 & 0,00 & 0,54 & 0,19 & $-0,82$ & 0,08 & 0,01 \\
\hline $\begin{array}{l}\text { PDA } \\
W 24\end{array}$ & 0,71 & $1-0,68$ & 0,16 & 0,08 & 0,00 & 0,66 & $-0,69$ & 0,30 & 0,06 & $-0,02$ & 0,67 & $-0,68$ & 0,29 & 0,05 & $-0,02$ \\
\hline $\begin{array}{l}\text { \%var. } \\
\text { Total }^{2} \\
\% \text { total } \\
\text { variance }\end{array}$ & 74,1 & 13,9 & 7,8 & 3,9 & 0,3 & 73,5 & 14,5 & 7,2 & 4,1 & 0,6 & 72,5 & 15,0 & 7,6 & 4,3 & 0,6 \\
\hline $\begin{array}{l}\text { GND } \\
D G B W\end{array}$ & $-0,04$ & $4-0,13$ & $-0,01$ & 0,99 & & $-0,10$ & 0,06 & $-0,22$ & 0,95 & & $-0,08$ & 0,06 & $-0,23$ & 0,97 & \\
\hline $\begin{array}{l}\text { GDA } \\
D G W 12\end{array}$ & 0,66 & $5 \quad 0,71$ & 0,20 & 0,12 & & 0,83 & $-0,49$ & 0,19 & 0,17 & & 0,78 & $-0,56$ & 0,22 & 0,15 & \\
\hline $\begin{array}{l}\text { GASA } \\
D G 1218\end{array}$ & $-0,40$ & 0,11 & 0,91 & 0,01 & & $-0,37$ & $-0,20$ & 0,88 & 0,23 & & $-0,40$ & $-0,15$ & 0,89 & 0,19 & \\
\hline $\begin{array}{l}\text { GSADA } \\
D G 1824\end{array}$ & 0,63 & $3-0,08$ & 0,36 & $-0,06$ & & 0,40 & 0,84 & 0,34 & 0,09 & & 0,40 & 0,81 & 0,33 & 0,07 & \\
\hline $\begin{array}{l}\text { \%var. } \\
\text { total } \\
\% \text { total } \\
\text { variance }\end{array}$ & 44,4 & 33,6 & 14,7 & 7,2 & & 43,8 & 32,7 & 14,6 & 8,9 & & 43,5 & $\mathbf{3 3 , 0}$ & 15,1 & 8,3 & \\
\hline $\begin{array}{l}1 \text { PN, PD, PA, PS } \\
\text { ganhos diários } \\
\text { respectivament } \\
\text { from birth to wean } \\
2 \text { Variância total }= \\
0,066 \text { (ganho) p }\end{array}$ & $\begin{array}{l}\text { SA, PD } \\
\text { de pes } \\
\text { te }(B W, \\
\text { ning, we } \\
=8262 \text {, }\end{array}$ & $\begin{array}{l}\text { A, GND } \\
\text { so do na } \\
\text { WW, } W 1 \\
\text { eaning to } \\
28 \text { (pes }\end{array}$ & $\begin{array}{l}\text {, GDA, } \\
\text { asciment } \\
12, \text { W18, } \\
12 \text { month } \\
\text { sos) e } 0,\end{array}$ & $\begin{array}{l}\text { ASA e } \\
\text { à desm } \\
4, D G B \\
12 \text { to } 18 \\
8 \text { (ganl }\end{array}$ & $\begin{array}{l}\text { E GS } \\
\text { nama, } \\
\text { 3W, DG } \\
\text { 8 mont } \\
\text { hos) }\end{array}$ & $\begin{array}{l}\text { são os } \\
\text { smam } \\
\text { of1218 } \\
18 \text { to } 2 \\
\text { mode }\end{array}$ & $\begin{array}{l}\text { s pesos } \\
\text { la aos } 12 \\
8, D G 182 \\
24 \text { months } \\
\text { lo I; } 294\end{array}$ & $\begin{array}{l}\text { o nasc } \\
\text { meses } \\
=\text { birth, } \\
\text { f age, } \\
\text {,24 ( }\end{array}$ & $\begin{array}{l}\text { mento } \\
\text { dos } 1 \\
\text { eaning } \\
\text { spectiv } \\
\text { so) e c }\end{array}$ & $\begin{array}{l}\text { à de } \\
\text { aos } \\
12,1 \\
l y) . \\
074\end{array}$ & $\begin{array}{l}\text { ha e } \\
\text { eses } \\
4 \text { mor } \\
\text { os) } p\end{array}$ & $\begin{array}{l}12,1 \\
\text { s } 18 \\
\text { of age } \\
\text { mo }\end{array}$ & $\begin{array}{l}24 \mathrm{~m} \\
24 \mathrm{n} \\
\text { ghts, } \\
\mathrm{II} ; 2\end{array}$ & $\begin{array}{l}\text { ses d } \\
\text { ses d } \\
\text { ily we } \\
5,55\end{array}$ & $\begin{array}{l}\text { dade, } \\
\text { dade, } \\
\text { tgains } \\
\text { eso) e }\end{array}$ \\
\hline
\end{tabular}

arquivo 2, toda a variância genética e parte da nãogenética; e no arquivo 3 , parte das variâncias genética e não-genética. Assim, de acordo com estes resultados, pode-se utilizar a variância fenotípica para o estudo dos componentes principais.

Para os pesos, a maior parte da variância total foi justificada pelo primeiro componente principal, que explicou 74,$1 ; 73,5$; e $72,5 \%$ para os três arquivos, respectivamente. Esse CP contrasta animais mais pesados com animais mais leves após o nascimento, e indica que a influência dos pesos se torna mais importante, à medida que o animal envelhece, como observaram BARBOSA e SMITH (1988) na raça Charolês.

Ainda para os pesos, o componente 2 explicou 13,$9 ; 14,5 ;$ e $15,0 \%$ da variância total para os três arquivos, respectivamente. Este CP descreve contrastes entre animais com maiores pesos da desmama aos 18 meses de idade com animais de maiores pesos aos 24 meses de idade, ou ainda, caracterizando animais com maior ou menor precocidade de crescimento e taxa de maturação precoce versus tardia. Os componentes 3,4 e 5 são todos ortogonais aos anteriores e entre si e seguem a mesma interpretação do componente 2 , ou seja, contrastam animais mais pesados em idades mais jovens com animais mais pesados em idades posteriores.

Para os ganhos de peso foram apresentados quatro componentes principais, sendo que o primeiro CP explicou 44,4; 43,8; e 43,5\% da variância total para os três arquivos, respectivamente. Esse CP contrastou animais com ganhos diferenciados em 
1660 Rev. bras. zootec.

diferentes épocas do ano; ou seja, o ganho ocorrido nas épocas desfavoráveis (desmama-ano e sobreano-dois anos) com ganho durante a época favorável (ano-sobreano), ou ainda, discriminou animais bons ganhadores de peso nas épocas favoráveis versus os bons ganhadores em épocas desfavoráveis. O segundo componente, que explicou 33,6; 32,7; e 33,0\% da variância total, respectivamente, não teve o mesmo comportamento para todos os arquivos. Considerando os arquivos II e III, observou-se contraste entre animais dentro da época de nascimento, ou seja, desmama-ano com sobreanodois anos e nascimento-desmama com ano-sobreano. Nos componentes 3 e 4, os ganhos dos 12 aos 18 meses e do nascimento à desmama foram as características que mais contribuem para os mesmos.

As correlações entre os componentes principais e as características consideradas reforçam as relações verificadas pelos componentes principais. As correlações do primeiro CP com os pesos da desmama aos 24 meses de idade foram positivas e altas, apresentando valores de 0,70;0,80;0,89; e 0,90 , respectivamente. As correlações entre o segundo CP com PD, PA e PDA foram medianas $(0,54 ; 0,43 ;$ e $-0,42$, respectivamente $)$. Estes valores indicaram os contrastes de animais que crescem mais rapidamente até o sobreano, com crescimento menos acelerado a partir dos dois anos. Para os ganhos de peso, o primeiro CP apresentou correlações altas com GDA $(0,84)$, GASA $(-0,59)$ e GSADA $(0,54)$, enquanto o segundo componente principal foi bem correlacionado com GDA $(-0,52)$ e GSADA $(0,81)$. Todas essas correlações estimadas foram significativas $(\mathrm{P}<0,001)$.

\section{Conclusões}

Com base nas estimativas de parâmetros genéticos obtidos, os melhores critérios de seleção para crescimento no Canchim foram os pesos aos 12 e 18 meses de idade ou os ganhos de peso da desmama aos 12 meses e dos 12 aos 18 meses de idade. A escolha de um ou outro dependerá das condições da fazenda e da época de nascimento.

Constatou-se, por meio dos resultados das análises de componentes principais, que a variação nos pesos e ganhos de peso foi bem sumarizada por três componentes principais, que envolveram, principalmente, os pesos da desmama aos 24 meses de idade e os ganhos de peso entre essas idades.

\section{Referências Bibliográficas}

ALENCAR, M.M., BARBOSA, P.F. 1982. Fatores que influenciam o peso de bezerros Canchim ao nascimento e à desmama. Pesq. Agropec. Bras., 17(10):1535-1540.

ALENCAR, M.M. 1985. Estudo da interação touro x época de nascimento. R. Soc. Bras. Zootec., 14(2):224-234.

ALENCAR, M.M., BARBOSA, P.F., BARBOSA, R.T. et al. 1993. Parâmetros genéticos para pesos e circunferência escrotal em touros da raça Canchim. R. Soc. Bras. Zootec., 22(4):572-583.

BAKER, J.F., STEWART, T.S., LONG,C.R. et al. 1988. Multiple regression and principal components analysis of puberty and growth in cattle. J. Anim. Sci., 66(9):2147-2158.

BARBOSA, P.F., SMITH, W.B. Aplicação de técnicas de análise multivariada em melhoramento animal. In: REUNIÃO ANUAL DA SOCIEDADE BRASILEIRA DE ZOOTECNIA, 25, 1988, Viçosa. Anais... Viçosa: SBZ, 1988, p.240.

BARBOSA, P.F., SILVA, A.H.G., PACKER, I.U. Causas de variação de pesos e ganhos de peso em fêmeas da raça Canchim. In: REUNIÃ̃ ANUAL DA SOCIEDADE BRASILEIRA DE ZOOTECNIA, 17, 1980, Fortaleza. Anais... Fortaleza: SBZ, 1980. p.167.

FREITAS, R., VENCOVSKY, R. Métodos de estimação de variâncias e parâmetros afins de características de crescimento em bovinos. In: REUNIÃO ANUAL DA SOCIEDADE BRASILEIRA DE ZOOTECNIA, 29, 1992, Lavras. Anais... LavrasL: SBZ, 1992. p.119.

MASCIOLI, A.S., ALENCAR, M.M., BARBOSA, P.F. et al 1996. Estimativas de parâmetros genéticos e proposição de critérios de seleção para pesos na raça Canchim. R. Soc. Bras. Zootec., 25(1):72-82.

MASCIOLI, A.S., PAZ, C.C.P., FARO, L. et al. 1997. Estimativas de parâmetros genéticos e fenotípicos para características de crescimento até a desmama em bovinos da raça Canchim. R. Soc. Bras. Zootec., 26(4):709-713.

OLIVEIRA, J.R. Estudo genético-quantitativo do desenvolvimento ponderal do gado Canchim. Ribeirão Preto: USP, 1979. 146p. Tese (Doutorado) - Escola Superior de Agricultura Luiz de Queiroz, 1979.

PACKER, I.U. Análise genética do crescimento até à desmama de bezerros Canchim. Piracicaba: USP, 1977. 173p. Tese (Livre Docência) - Escola Superior de Agricultura Luiz de Queiroz, 1977.

ROSO, V.M., FRIES, L.A. 1995. Componentes principais em bovinos da raça Polled Hereford à desmama e sobreano. R. Soc. Bras. Zootec., 24(5):728-735.

SAS statistical analysis system user's guide: Stat, Version 6.11 . Cary: SAS Institute, 1996.

SILVA, A.H.G., PACKER, I.U., BARBOSA, P.F. Heritability estimates for pre and pos weaning weights of Canchim calves. In: CONFERÊNCIA INTERNACIONAL DE BIOMETRIA, 10, 1979, Guarujá. Anais... Brasília: EMBRAPA-DID.DMQ, 1982. p.101.

Recebido em: 08/03/99

Aceito em: 19/06/00 